\title{
Article \\ Image Encryption Based on Arnod Transform and Fractional Chaotic
}

\author{
Chao Chen ${ }^{1,2}$, Hongying Zhang ${ }^{1}$ and Bin $\mathrm{Wu}^{1, *}$ \\ 1 School of Information Engineering, Southwest University of Science and Technology, \\ Mianyang 621010, China; ch10503@126.com (C.C.); zhywyd@163.com (H.Z.) \\ 2 Data Recovery Key Laboratory of Sichuan Province, Neijiang Normal University, Neijiang 641100, China \\ * Correspondence: wubin@swust.edu.cn
}

Citation: Chen, C.; Zhang, H.; Wu, B. Image Encryption Based on Arnod Transform and Fractional Chaotic. Symmetry 2022, 14, 174. https:// doi.org/10.3390/sym14010174

Academic Editors: Liang-Jian Deng Xian-Ming Gu and Tai-Xiang Jiang

Received: 14 October 2021

Accepted: 5 January 2022

Published: 17 January 2022

Publisher's Note: MDPI stays neutral with regard to jurisdictional claims in published maps and institutional affiliations.

Copyright: (c) 2022 by the authors. Licensee MDPI, Basel, Switzerland. This article is an open access article distributed under the terms and conditions of the Creative Commons Attribution (CC BY) license (https:/ / creativecommons.org/licenses/by/ $4.0 /)$.

\begin{abstract}
An image encryption and decryption algorithm based on Arnod transform and fractional chaos is proposed in this work for solving the problem that the encrypted image is easily cracked and the content of the decrypted image is distorted. To begin with, the Arnold transform is used to encrypt, so that the spatial confidence of the original image has been comprehensively disturbed. Secondly, the XOR involving the fractional order chaotic sequence is used to encrypt. The key sequence is dynamically generated to ensure the randomness and difference of key generation. When decryption is required, the first decryption is performed using the key and XOR. Then, the second decryption is carried out by using the inverse Arnold transform, and finally the decrypted image is obtained. Experimental results show that the improved algorithm has achieved a better performance in encryption and decryption.
\end{abstract}

Keywords: fractional derivative; Arnold transform; XOR involving fractional order chaotic sequence; encryption and decryption

\section{Introduction}

With the popularity of smart phones, image encryption in the process of transmission has become an important research topic. The security of image transmission is closely related to personal privacy and property safety. It seems to be somewhat inadequate by relying on only legal constraints. Li X, Zhou put forward the idea of DNA and space-time chaos, which made good use of the coding characteristics of DNA to encrypt images, and achieved good results [1]. Liu $\mathrm{H}$ and Jin proposed encrypting images based on Arnold transform, quantum chaos and the S-Box model [2,3]. Quantum chaos spreads the degree of scrambling of plaintext well and achieves a better encryption effect. Some scholars also used $\mathrm{YCbCr}$ space to encrypt images [4] and converted to color images to encrypt images. It makes good use of the effect of image stratification. Ni Z and Kang improved the Arnold transformation and quantum chaos [5,6]. With a very good combination of each other's advantages, the encryption effect is remarkable. Xu L, Gou and others put forward an image encryption algorithm based on improved standard mapping, and used a function to map the image encryption algorithm, and achieved remarkable results [7]. Vaish A, Kumar used MSVD, DWT and Arnold transformed to encrypt images [8], which effectively prevented the hacker's violent attack. Chai X and Gan Z. put forward the compress perception to encrypt images combined with the related concepts of physics, and the encryption effect was good [9]. Domestic scholars put forward the research of Arnold transform and Gray code transformation, the blind detection robust digital watermark embedding strategy, the combination of Tetrolet transform and SVD, the digital image encryption algorithm based on Arnold hierarchical cyclic transformation, and used Logistic and Rossler to encrypt the image [10-14]. Gong Lihua introduced a fractional partial derivative [15] converted to a frequency domain filter and combined two dimensional Arnold transform to encrypt an image, so that the image was encrypted in both spatial and frequency domains, and a good 
encryption effect was obtained. However, the method is simple and easily attacked by hackers. In recent years, skew tent chaos mapping combined with Arnold transform has been proposed to encrypt images by means of enhanced singular value decomposition and zero watermark of a cellular neural network [16-18]. However, this only changes the spatial location information of pixels. After finding the Arnold Transform inverse function, the key can be quickly obtained. Good results have been achieved in encryption, but there were still some flaws in the hacker's violent decryption of the encrypted image. In view of the fact that image encryption is not absolutely safe and partly distorted after image restoration. An image encryption and decryption algorithm based on Arnold transform is put forward to disturb the texture of the image and spatial pixel distribution. Then, it uses the XOR involving the fractional order chaotic sequence to change the pixel value itself. After two encryptions, the difficulty of hacking is greatly enhanced. After receiving the image, the information of the original image can be obtained after two decryptions. Experimental results have shown that the improved algorithm achieves better results, and the security and difficulty of image transmission are greatly improved. It is difficult to decipher the real data of the image without obtaining the random fractional derivative sequence.

\section{Preliminary}

\subsection{The Principle of Image Encryption Using Arnold Transform}

The essence of Arnold transform is used to change the coordinates of original pixels in the spatial domain. Arnold transformation is also commonly known as "cat-facet transformation" [3]. The specific two-dimensional Arnold transformation formula can be written as:

$$
\left[\begin{array}{l}
x^{\prime} \\
y^{\prime}
\end{array}\right]=\left[\begin{array}{ll}
1 & 1 \\
1 & 2
\end{array}\right]\left[\begin{array}{l}
x \\
y
\end{array}\right]
$$

where: $(x, y)$ is the spatial 2-dimensional coordinates of the pixel located in the image to be encrypted $\left(x^{\prime}, y^{\prime}\right)$ is 2-dimensional coordinates transformed after the cat is transformed. The Arnold transform matrix is symmetrical. Using the Arnold transform to encrypt the image basing on first encryption, it became chaotic in space, so as to achieve the effect of searching for regularity when disturbing hacker attacks.

\subsection{First Image Encryption Principle of Arnold Transform}

Arnold Transformation has periodicity [11,12], and periodicity is not proportional to the size of the image. Table 1 shows the relationship between the order of the image and the period [15-18]:

Table 1. Relationship between the period of Arnold transformation and the number of images.

\begin{tabular}{cccc}
\hline Order & Period & Order & Period \\
\hline 4 & 3 & 64 & 48 \\
8 & 6 & 128 & 96 \\
16 & 12 & 256 & 62 \\
32 & 24 & 512 & 384 \\
\hline
\end{tabular}

All the image sizes to be encrypted in this experiment are normalized to $128 \times 128$. According to the relationship between the Arnold transform cycle and the order of image displayed in Table 1, the Arnold transform period of this experiment was 96. The Arnold transform encrypted image can recover image original data by inverse operation.

\section{Analysis of Proposed Method}

\subsection{Improving the Principle of Image Encryption and Decryption}

The improving two-dimensional Arnold transformation formula can be written as

$$
\left[\begin{array}{l}
x^{\prime} \\
y^{\prime}
\end{array}\right]=\left[\begin{array}{c}
1 a \\
b a b+1
\end{array}\right]\left[\begin{array}{l}
x \\
y
\end{array}\right]
$$


where:

$$
\begin{gathered}
a=\operatorname{ceil}\left(\operatorname{mean}\left(u_{(0)}+\frac{\mu}{\Gamma(v)} \sum_{j=1}^{n} \frac{\Gamma(n-j+v)}{\Gamma(n-j+1)} u(j-1)(1-u(j-1))\right)\right) \\
b=\operatorname{ceil}\left(\operatorname{mean}\left(u_{(0)}{ }^{\prime}+\frac{\mu^{\prime}}{\Gamma(v)} \sum_{j=1}^{n} \frac{\Gamma(n-j+v)}{\Gamma(n-j+1)} u^{\prime}(j-1)\left(1-u^{\prime}(j-1)\right)\right)\right),
\end{gathered}
$$

where mean is the mean values of the generated random sequences, ceil is an integer round up. The parameters in Equation (3) are:

$$
v=0.7, \mu=2.4, \mu(0)=0.1 \text {. }
$$

The parameters in Equation (4) are:

$$
v=0.8, \mu=2.5, \mu(0)=0.2 \text {. }
$$

in Equation (3) and in Equation (4) they were brought into Equation (2). Finally, a transformation matrix (in Equation (2)) is obtained.

\subsection{Arnold Transformation Using the Fractional Logistic Map}

The logistic map is a polynomial mapping (equivalently, recurrence relation) of degree 2 , which is often cited as an archetypal example of complex, so that chaotic behavior can arise from very simple non-linear dynamical equations. The map was popularized in a seminal 1976 paper by the biologist Robert May, in part as a discrete-time demographic model analogous to the logistic equation first created by Pierre François Verhulst. Onedimensional Logistic mapping is the most widely applied chaotic mapping currently; the chaotic models generated by it are also known as the insect amount model, and is mathematically defined as follows [16,17]

$$
\mu_{n+1}=\mu_{0}+\mu_{n}\left(1-\mu_{n}\right)
$$

The dynamic state of logistic mapping is chaotic. At this time, the chaotic sequence generated by logistic mapping has significant non-periodicity and non-convergence. Many hackers are familiar with this conventional encryption method, so this encryption method is relatively mature and easy to crack, so that the security of the image encryption method cannot be guaranteed.

Inspired by Guocheng Wu's related literature [19-22], non-Fourier's heat flux and non-Fick's mass flux theory had been used to save the numerical study of bioconvection flow of nanofluids [23-27]. We introduce the fractional order chaotic sequence to improve the Arnold transform.

$$
u_{(n)}=u_{(0)}+\frac{\mu}{\Gamma(v)} \sum_{j=1}^{n} \frac{\Gamma(n-j+v)}{\Gamma(n-j+1)} u(j-1)(1-u(j-1)),
$$

where $\Gamma$ is Gamma function, $v$ is fractional order. The stability, turbulence and chaos of Equation (6) can be discussed in reference to $[19,20,26]$.

\subsection{Arnold Transformation Using the Fractional Logistic Map}

Research shows that when the determinant value of the transformation matrix $\left[\begin{array}{c}1 a \\ b a b+1\end{array}\right]$ is 1 , the Arnold map enters a chaotic state. However, there is a one to one relationship between the spatial coordinates before and after transformation, and the periodic $\mathrm{T}$ before Arnold will be extended indefinitely. After $n$ times of Arnold transformation, the image scrambling effect is very good, the distribution of image pixels is fairly uniform, and the irrelevance between pixels is enhanced to achieve a good encryption effect. Based on 
the periodicity and reversibility of Arnold transform and the randomness of a fractional chaotic sequence, a color digital image encryption algorithm based on Arnold transform is proposed. Through the improved Arnold transform, the image is encrypted by disorderly and distinct images, and it is difficult to correlate with the original image. The improved method not only simplifies the image encryption and decryption operations, but also does not add additional storage space. Theoretically, it can restore the encrypted image as completely lossless.

\subsection{Fractional Order Logistic Chaos XOR}

XOR algorithm principle: The XOR operation has a curious feature: if you do XOR twice in a row to a value, it returns the value itself. The XOR operation is most suitable for simple encryption and decryption. The principle of this method is that when the XOR operation is performed on one number $\mathrm{A}$ and another number $\mathrm{B}$, another number $\mathrm{C}$ will be generated. If the XOR operation is performed on Key and B, then $C$ will be restored to A. Compared with other simple encryption algorithms, the XOR algorithm has many advantages, such as the algorithm being simple, fast and the encryption of the gray value of the image can achieve complete lossless reduction.

We randomly select a sequence from the three fractional order chaotic sequences as the XOR encryption key. On the basis of the first use of the Arnold transform, the XOR operation is used for secondary encryption to enhance the security of image encryption again.

$$
\operatorname{Key}=\operatorname{ceil}\left(\operatorname{mean}\left(u_{(0)^{\prime \prime}}+\frac{\mu^{\prime \prime}}{\Gamma(v)} \sum_{j=1}^{n} \frac{\Gamma(n-j+v)}{\Gamma(n-j+1)} u^{\prime \prime}(j-1)\left(1-u^{\prime \prime}(j-1)\right)\right)\right) \text {. }
$$

\section{Implementation}

The image contains R, G, B, three primary colors, respectively, and encryption and decryption of the three primary colors. This paper only deals with the two level decomposition of encrypted color digital images. The above methods are used to encrypt the $G$ and B primary color matrix of the image. Finally, the encrypted R, G and B primaries are combined to obtain the encrypted color digital images and the Arnold inverse transform for the current image. Because the Arnold transform is periodic, that is to say, assuming that the Arnold period of an image is T, the original image is scrambled by $\mathrm{T}$ times Arnold transform and then returns to the original image. Therefore, if the number of scrambling of the Arnold transform is $k$ when the original R-base color is encrypted, then the encrypted Rbase color is re-transformed by the T-k Arnold transform, and the unencrypted R-base color is returned, that is to say, the decryption effect is achieved, and a satisfactory decryption effect can be achieved. For the encrypted images, the $\mathrm{G}$ and $\mathrm{B}$ primary color matrices are encrypted by the above methods. Finally, the decrypted R, G and B primaries are combined to obtain the deciphered color digital images. The procedure of our proposed algorithm is as follows. The two imported original images are the input of our proposed algorithm.

Step 0: Three fractional order chaotic sequences are generated and two are chosen randomly as $a, b$ in the Arnold transform matrix.

Step 1: Using fractional order Logistic chaos Arnold transform to realize encryption of spatial coordinates;

Step 2: Three fractional order chaotic sequences are generated and one is chosen randomly as key. The parameters in this key are: $v=0.9, \mu=2.7, \mu(0)=0.3$.

Step 3: Using XOR involving key to realize encryption.

Step 4: Taking the encrypted image with the key (fractional order logistic chaotic sequence to) to decrypt the image.

Step 5: Using Arnold inverse transformation to realize second encryption of spatial coordinates.

Finally, the result of the output is a decrypted complete image. 


\section{Experiment and Performance Comparison}

\subsection{Distribution Characteristic of Pixel Value}

The histogram of the plaintext image and the histogram of the ciphertext image are very different, so it cannot reflect the pixel distribution characteristics of the image. Deliberately stitching the opponent's image and scrambling spatial coordinates will effectively confuse the hacker against the use of statistical gray value distribution to attack encrypted images. The experimental results show that there are great differences in the histogram before and after scrambling.

\subsection{Correlation Analysis of Each Other Image}

The adjacent pairs of pixels in horizontal, vertical and diagonal directions are calculated by Equation (8).

$$
r=\frac{\sum_{i=1}^{M_{0}}\left(x_{i}-\bar{x}\right)\left(y_{i}-\bar{y}\right)}{\sqrt{\sum_{i=1}^{M_{0}}\left(x_{i}-\bar{x}\right)^{2}\left(y_{i}-\bar{y}\right)^{2}}} .
$$

The two pixel values of the adjacent pixels $X_{i}$ and $Y_{i}$ of group $i$ in the image, $x, y$ are the mean values of pixel values $X_{i}$ and $Y_{i}$, respectively; $M$ is the neighbor pixel. The improved algorithm can be very good at disordering the correlation between pixels.

\subsection{Analysis of the Ability to Resist the Difference Attack}

NPCR: Number of Pixels Change Rate. It measures the sensitivity of encryption algorithm to plaintext [14-18]; UACI: Unified Average Changing Intensity measures the sensitivity of encryption [14-18]. When there is only one pixel in the process of encryption and decryption, set the pixel values of their $(i, j)$ points in their ciphertext images respectively $C_{1(i, j)}$ and $C_{2(i, j)}$. If $C_{1(i, j)}=C_{2(i, j)}$, define $D_{(i, j)}=0$; If $C_{1(i, j)} \neq C_{2(i, j)}$, define $D_{(i, j)}$. So, formulas for the calculation of NPCR are as follows [14-18]:

$$
\begin{gathered}
\mathrm{NPCR}=\frac{1}{M * N} \sum_{i=1}^{M} \sum_{j=1}^{N} D(i, j) * 100 \% \\
\mathrm{UACI}=\frac{1}{M * N} \sum_{i=1}^{M} \sum_{j=1}^{N} \frac{\left|c_{1}(i, j)-c_{2}(i, j)\right|}{255} * 100 \% .
\end{gathered}
$$

The expected value of the two is calculated by using the Equations (11) and (12) [14-18]:

$$
\begin{gathered}
\mathrm{NPCR}_{E}=\left(1-2^{-n}\right) \times 100 \% \\
\mathrm{UACI}_{E}=\frac{1}{2^{2 n}} \frac{\sum_{i=1}^{2^{n}-1} i *(i+1)}{2^{n}-1} \times 100 \% .
\end{gathered}
$$

There are $M$ pixels in the vertical direction of the image and $N$ pixels in the horizontal direction of the image, which can test the sensitivity before and after encryption.

\subsection{Information Entropy Analysis}

Using Equation (13) to calculate information entropy, which is an important measure to reflect the randomness of information [14-18]:

$$
H(S)=-\sum_{i=0}^{2^{n}-1} P\left(s_{i}\right) \log 2\left(P\left(s_{i}\right)\right),
$$


where: $P\left(s_{i}\right)$ represented the probability of the emergence of pixel $S_{i}, 2^{n}$ represented the number of states that appear in total, $H(S)$ can test the amount of information covered by encrypted images and pre-encrypted images.

\subsection{Abbreviatio of the Relative Comparative Experimental Data}

To simplify the representation, we simplify the relevant parameters here. Hm1: The information entropy of the original image. Hm2: Information entropy of encrypted image, NPCR: Pixel number change rate, UACI:Normalized pixel value average change intensity.

\subsection{Detailed Experimental Data}

\section{(a) First Group Contrastive Experiment}

The original image, encrypted image and decrypted image of the Lena image are shown in Figure 1, respectively.
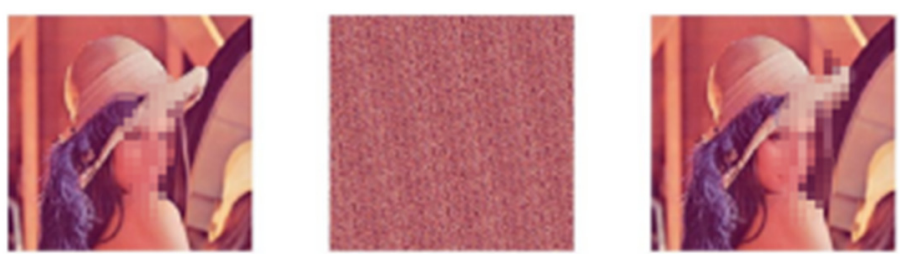

Figure 1. Comparison map before and after encryption of the Lena.

We can see that the encrypted image is completely misplaced in space, and there is no connection between the encrypted image and the original Lena image. The decrypted Lena image has no change with the original image. Three color RGB channels' s histogram of the original Lena image is shown in Figure 2.
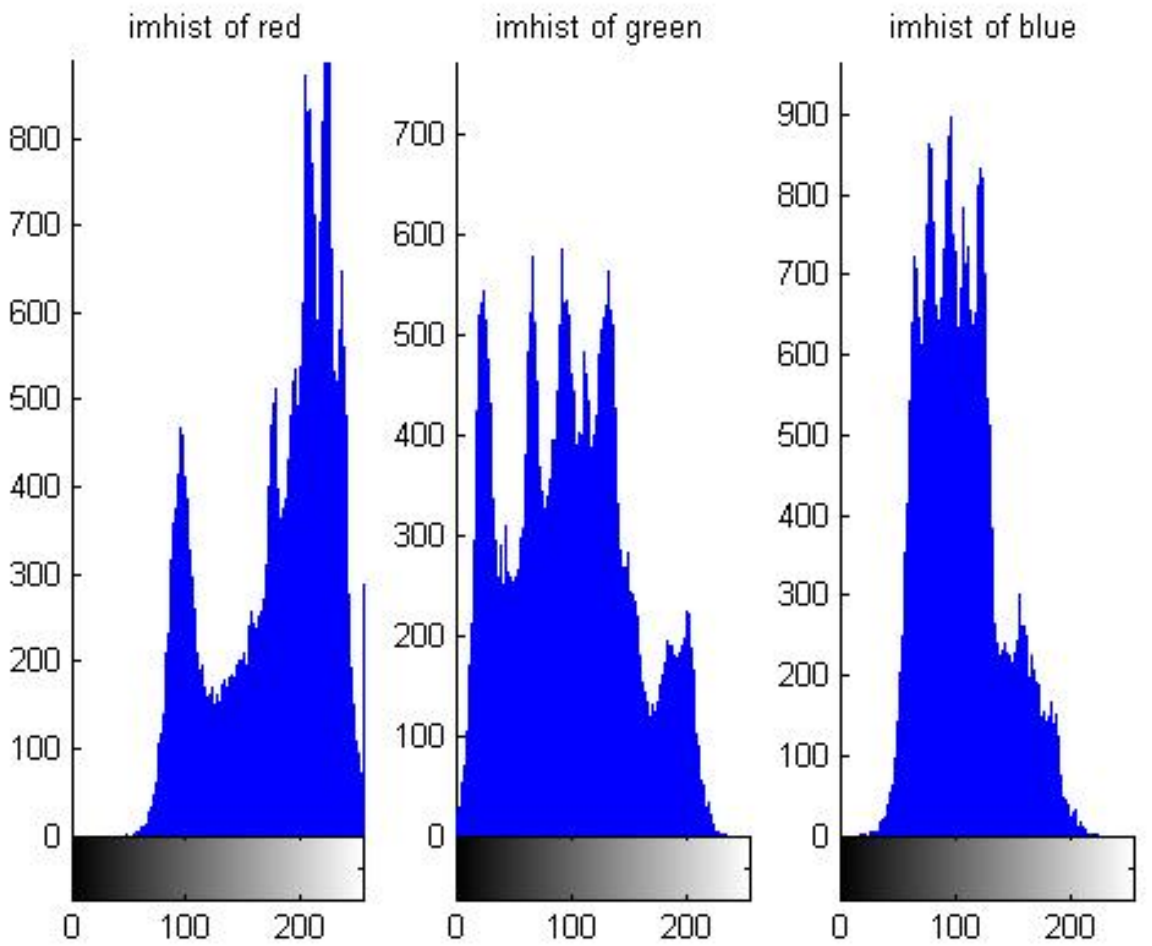

Figure 2. Histogram of original Lena image.

Three color RGB channels' histogram of Lena' s encrypted image is shown in Figure 3. 

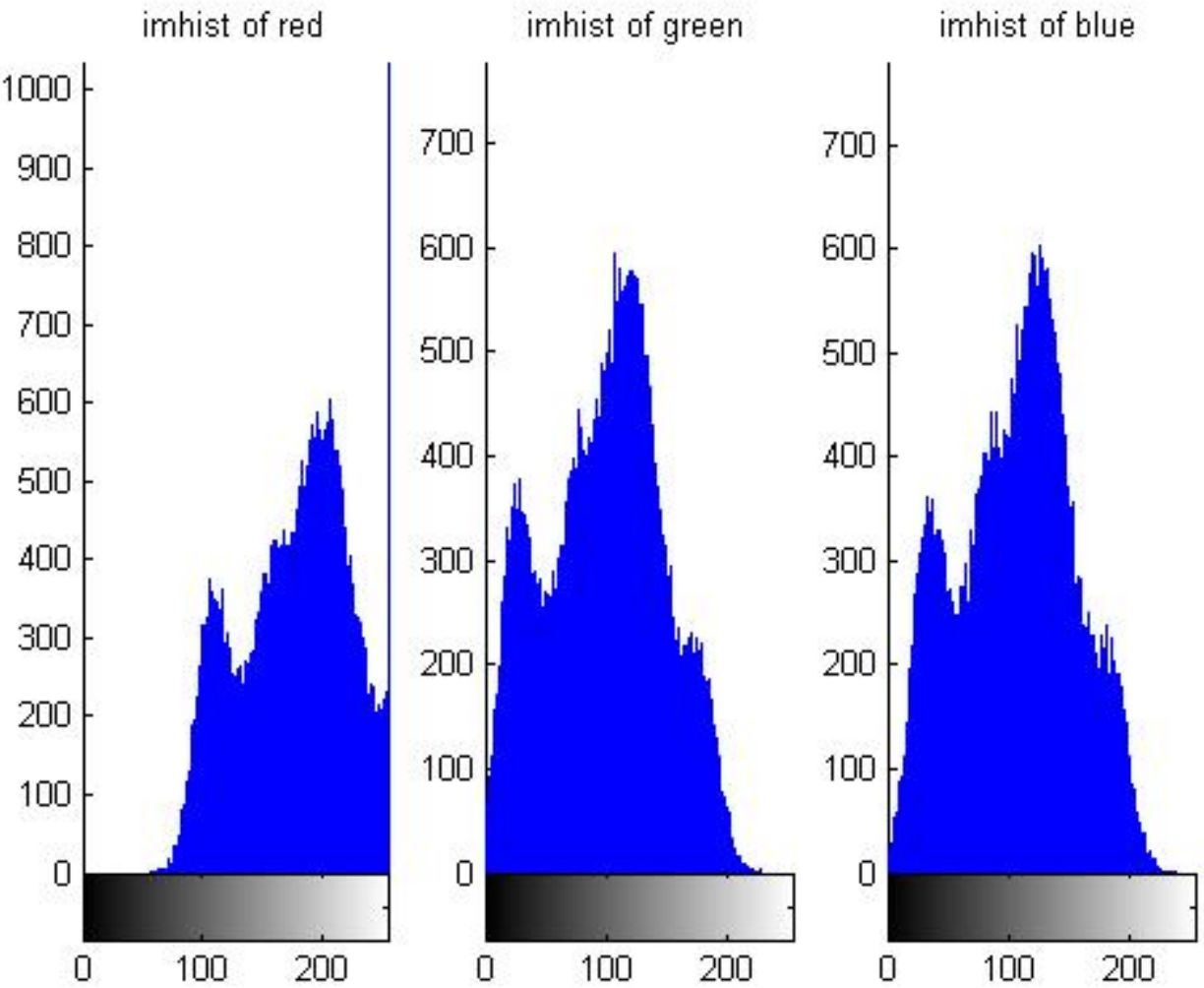

Figure 3. Histogram of encrypted Lena image.

It can be seen that the histogram has undergone a large transformation and can hardly reflect the gray value distribution of the three channels. A correlation analysis of the original image and the encrypted image is shown in Figure 4.
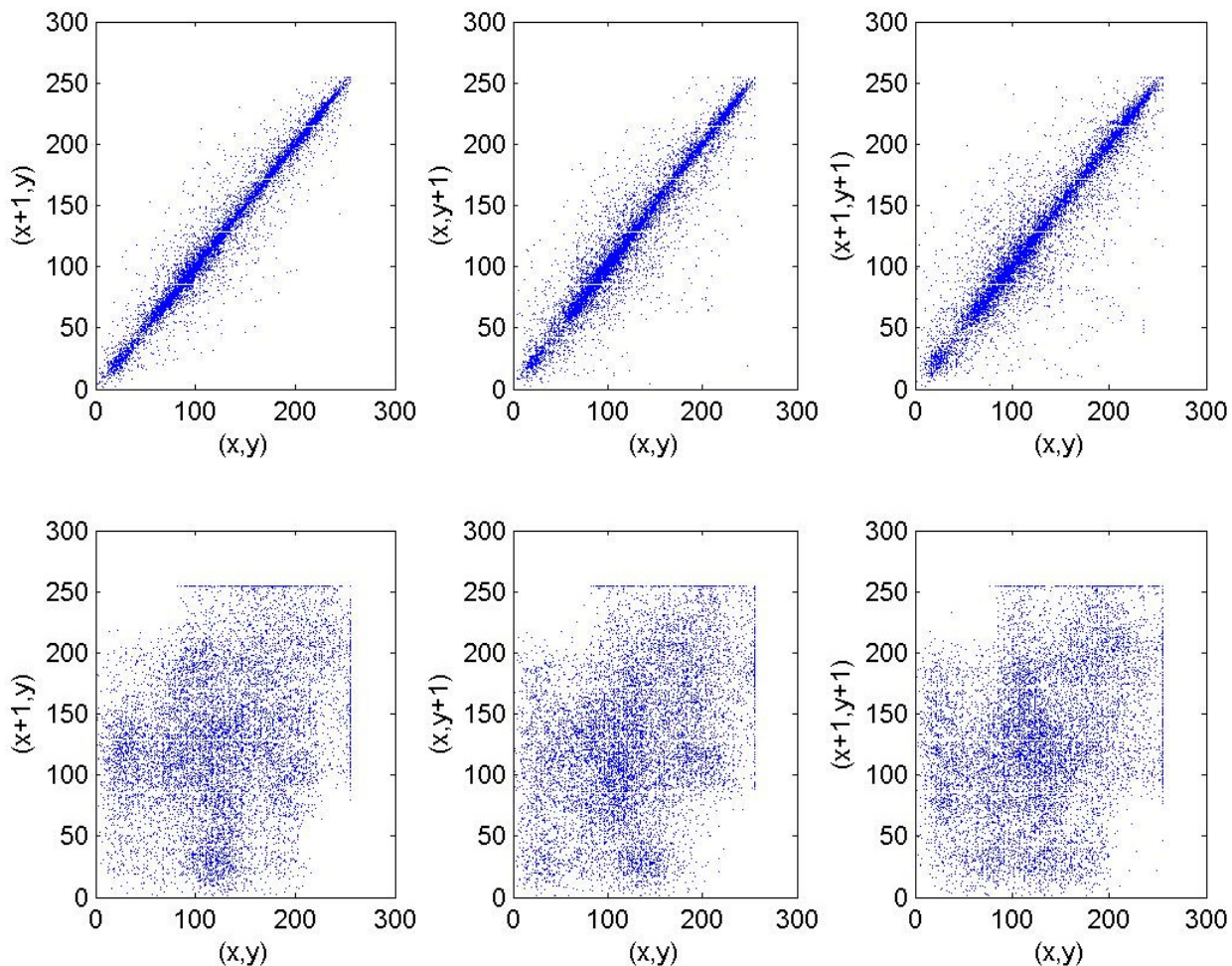

Figure 4. Correlation charts of three directions before and after encrypted Lena image. 
The encrypted image is well scrambled in three directions. The details of an analysis of the ability to resist the difference attack are shown in Table 2.

Table 2. Lena's information entropy and NPCR, UACI.

\begin{tabular}{ccccc}
\hline & Hm1 & Hm2 & NPCR & UACI \\
\hline Lena & 7.7580 & 7.7816 & 0.1723 & 0.0725 \\
\hline
\end{tabular}

As can be seen from Table 2, the encryption effect is good, and it can withstand the attack of hackers well.

\section{(b) Second Group Contrastive Experiment}

The original image, encrypted image, and decrypted image of the girl image are shown in Figure 5, respectively.
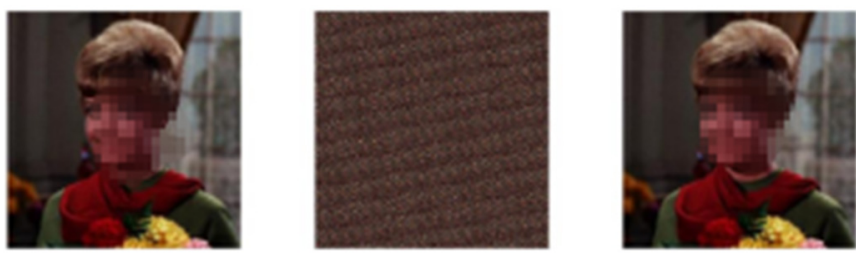

Figure 5. Comparison map before and after encryption of the girl.

The encrypted image is a mess. We can see that the encrypted image is fundamentally not related to the original image. The three color RGB channels' histogram of the girl's encrypted image is shown in Figures 6 and 7.
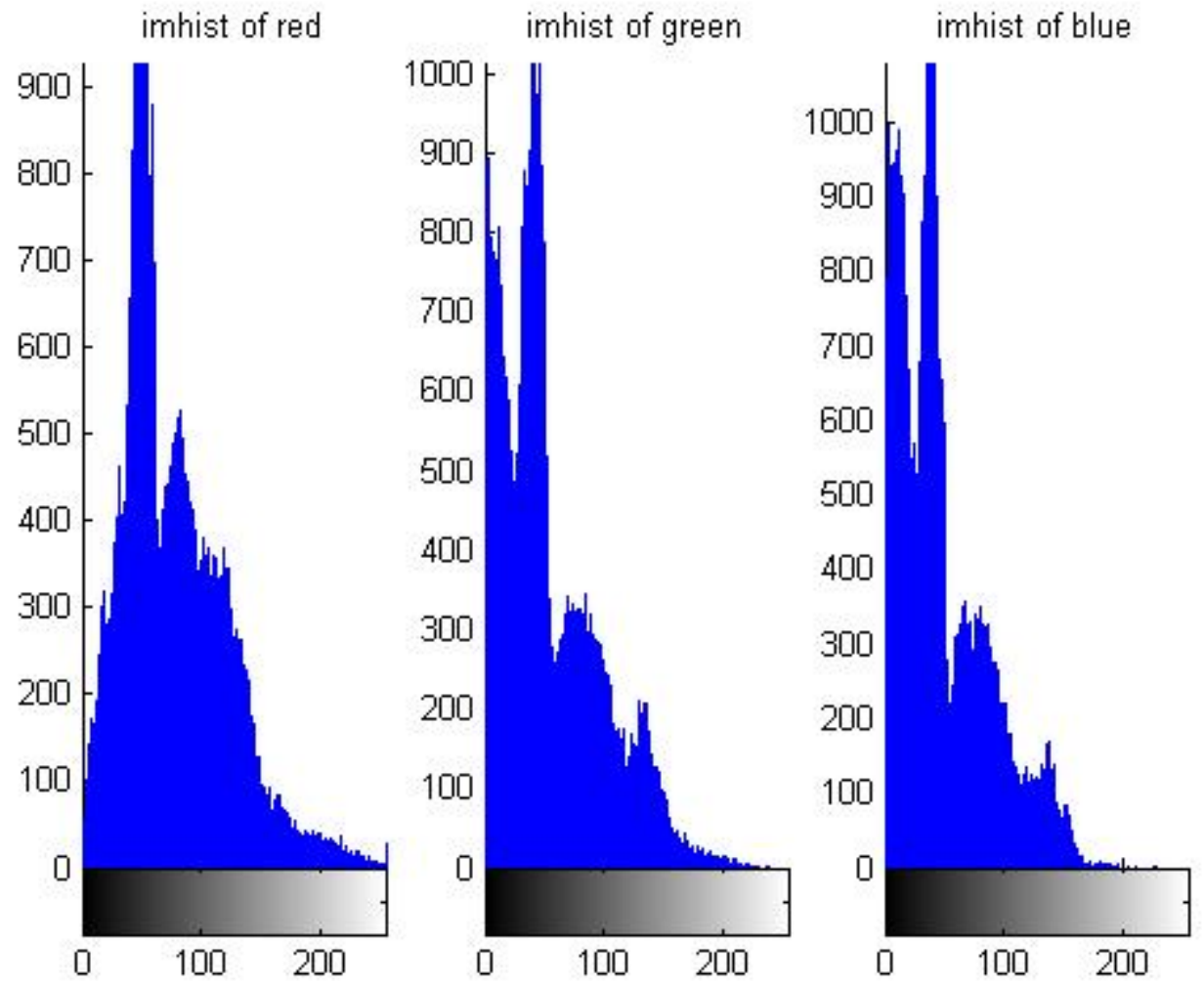

Figure 6. Histogram of original girl image. 

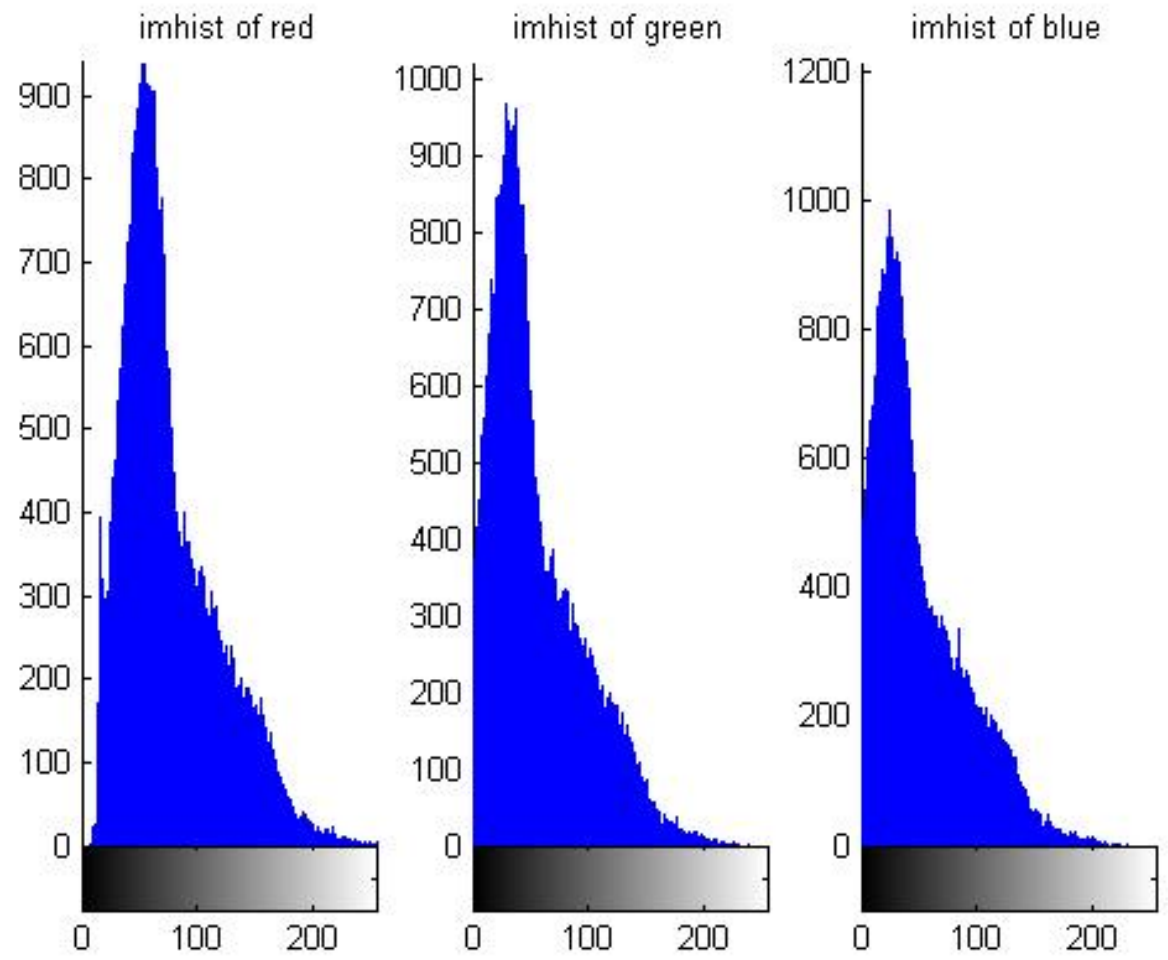

Figure 7. Histogram of encrypted girl image.

From the histogram information, we can know that the gray value distribution of the image changes obviously, which can disturb the hacker's feature analysis very well. Three directions' correlations before and after encryption are shown in Figure 8.
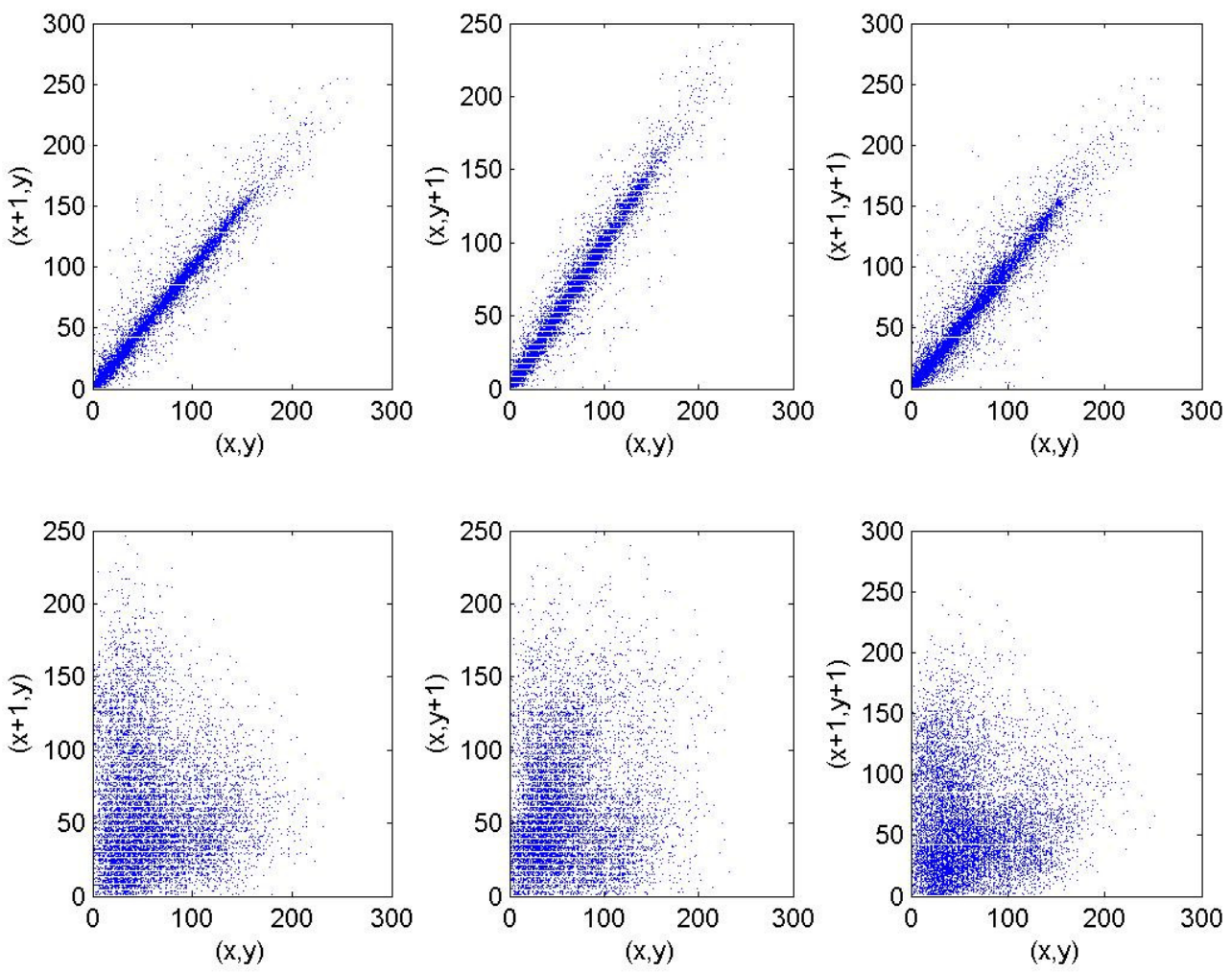

Figure 8. Correlation charts of three directions before and after encrypted girl image. 
We disturb the correlation analysis of hackers from three directions and analyze the ability to resist the difference attack. The details are shown in Table 3.

Table 3. Girl' s information entropy and NPCR, UACI.

\begin{tabular}{ccccc}
\hline & Hm1 & Hm2 & NPCR & UACI \\
\hline girl & 7.0467 & 6.9732 & 0.1638 & 0.0586 \\
\hline
\end{tabular}

(c) Third Group Contrastive Experiment

The original image, encrypted image, and decrypted image of the peppers image are shown in Figure 9, respectively.
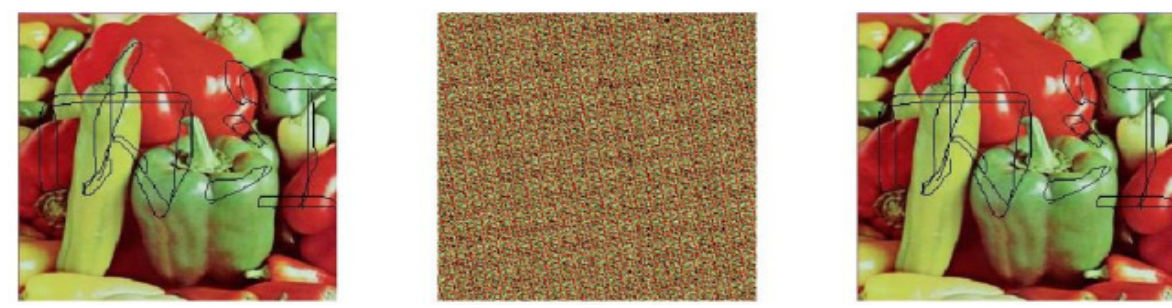

Figure 9. Comparison map before and after encryption of the peppers. The Chinese character "Neijiang" is the name of a prefecture-level city in China.

In the first image of Figure 9, only one pixel wide text is scrambled, and after decryption, the original image is completely restored. The three color RGB channels' histogram of the pepper's encrypted image is shown in Figures 10 and 11.
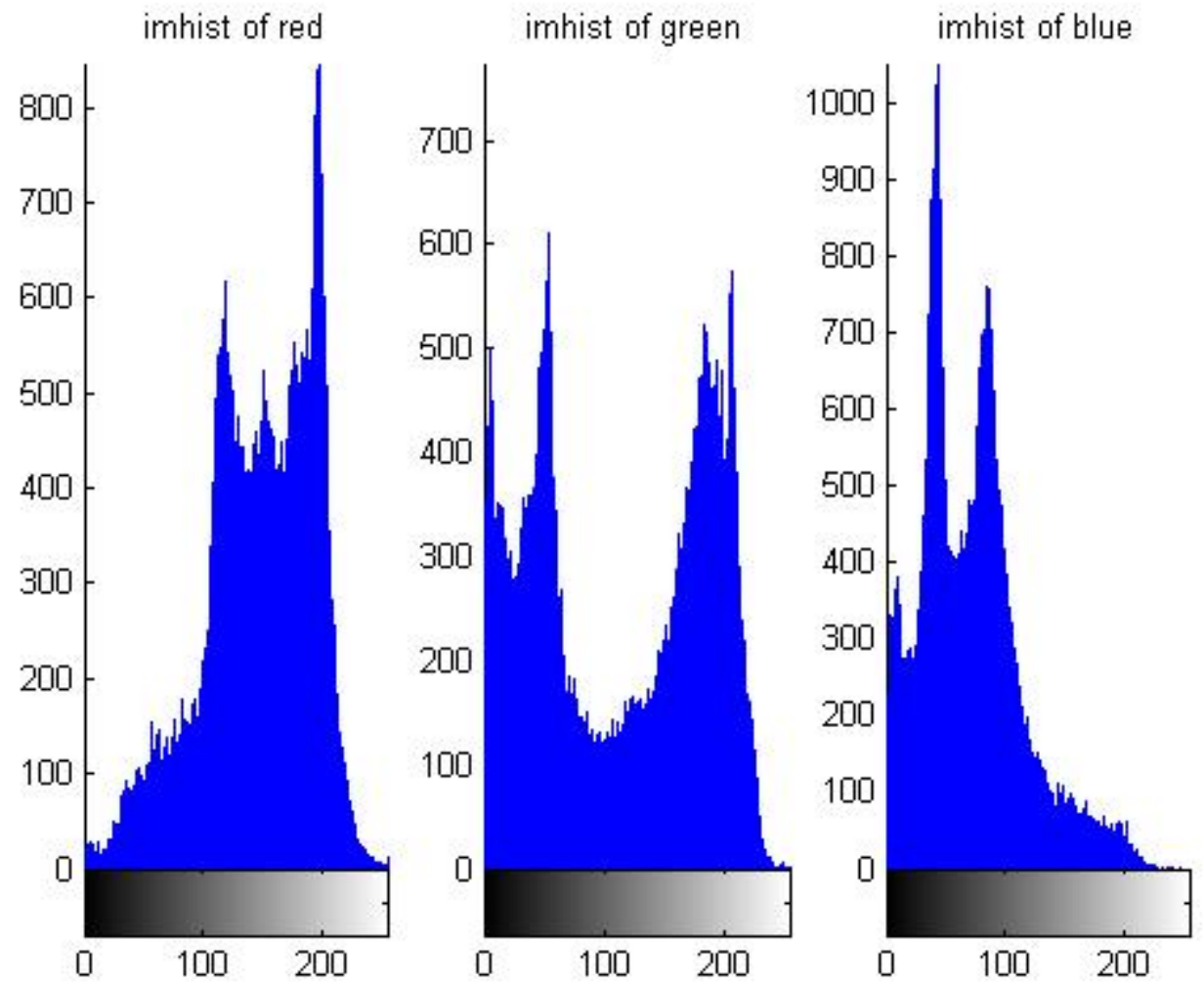

Figure 10. Histogram of original peppers image. 

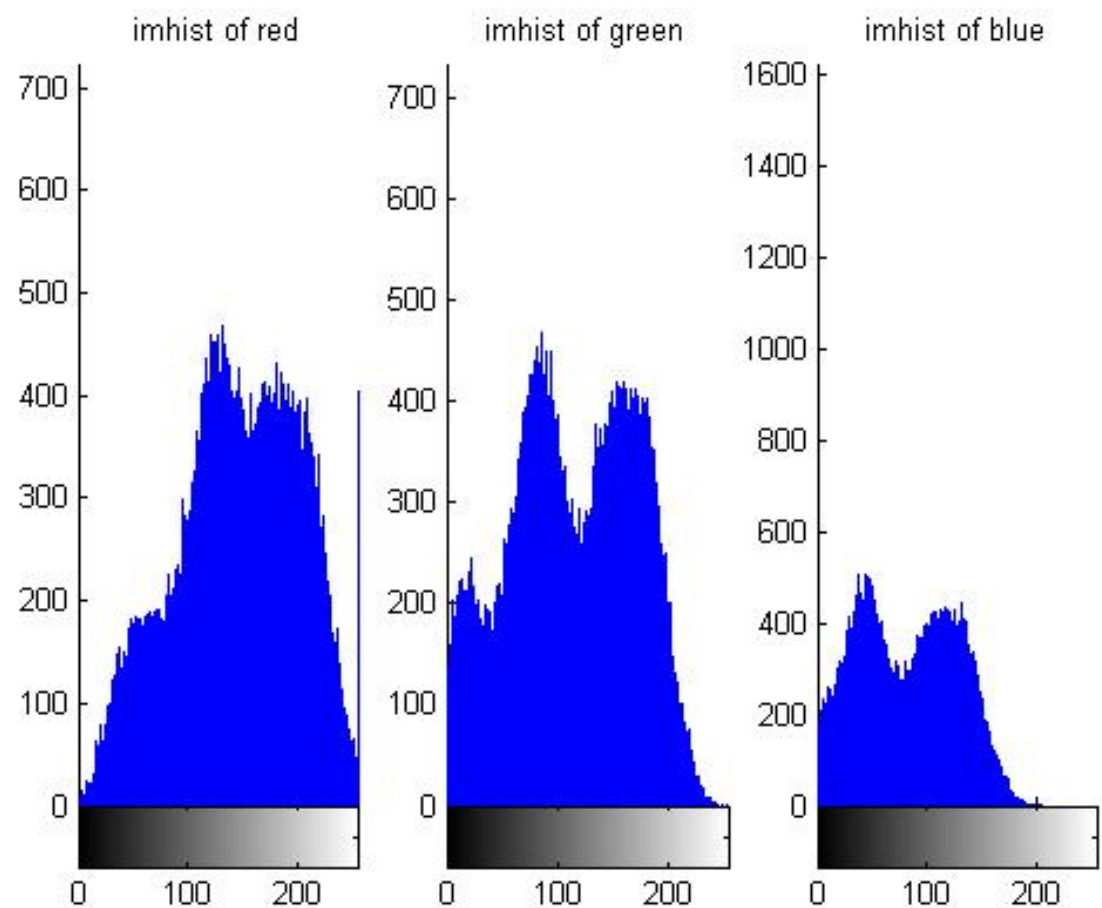

Figure 11. Histogram of encrypted peppers image.

From the histogram information, we can know that the histogram information has been greatly changed, which can increase the difficulty of cracking encrypted images. In particular, the feature distribution of the red channel and the green channel changed greatly. However, the histogram, as an important analysis meaning to crack image encryption, can well prevent crackers from analyzing image features. Three directions' correlation before and after encryption are shown in Figure 12.
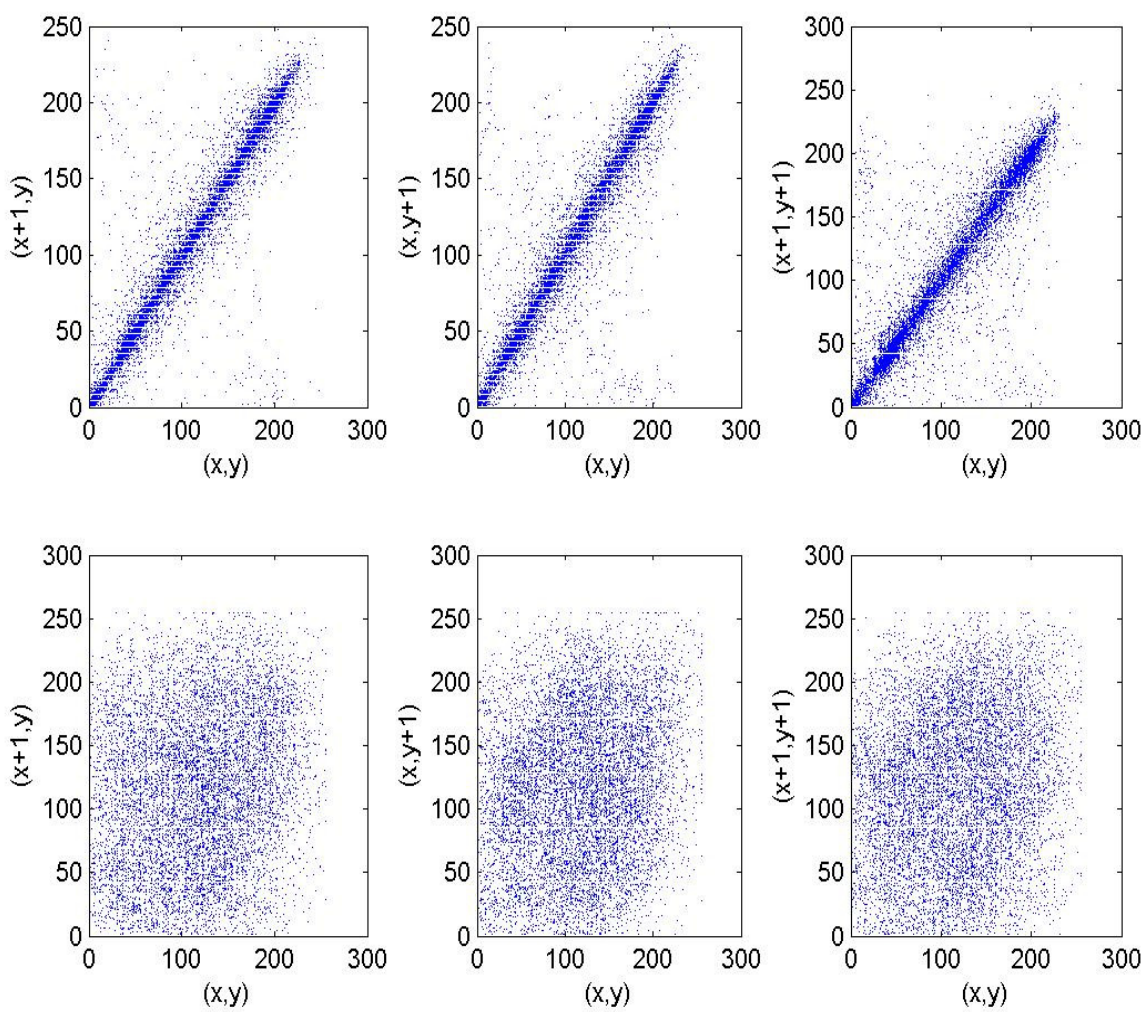

Figure 12. Correlation charts of three directions before and after encrypted peppers image. 
The correlation between the three directions indicates the degree of correlation between them. As for the analysis of the ability to resist the difference attack, the details are shown in Table 4.

Table 4. Peppers' information entropy and NPCR, UACI.

\begin{tabular}{ccccc}
\hline & Hm1 & Hm2 & NPCR & UACI \\
\hline peppers & 7.5743 & 7.4929 & 0.1661 & 0.0757 \\
\hline
\end{tabular}

Information Entropy can well express how much information the image contains. As long as there are some differences, it will increase many difficulties in cracking the content of image encryption.

\section{(d) Fourth Group Contrastive Experiment}

For animals, hair is the most important feature, and the basic five features are embedded in it. Here, our algorithm can integrate these features very well, especially the number of pixels in it is also well encrypted, which is difficult to crack through conventional algorithms. Using the corresponding inverse transform, the image information can be restored completely. The original image, encrypted image, and decrypted image of the pepper's image are shown in Figure 13, respectively.
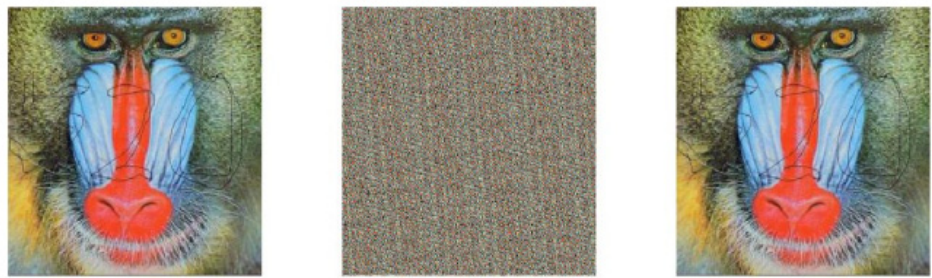

Figure 13. Comparison map before and after encryption of the baboon.

The encrypted image presents complete confusion, which has caused more obstacles to the cracked person. The three color RGB channels' histogram of the baboon's encrypted image is shown in Figures 14 and 15.
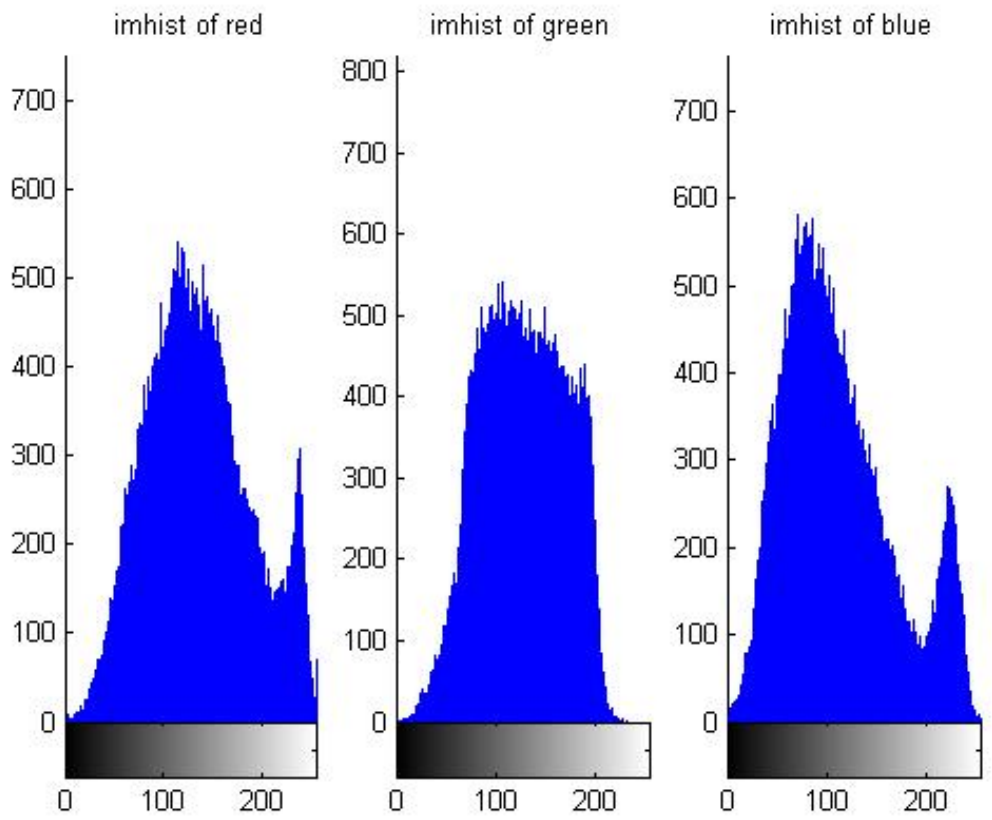

Figure 14. Histogram of original baboon image. 

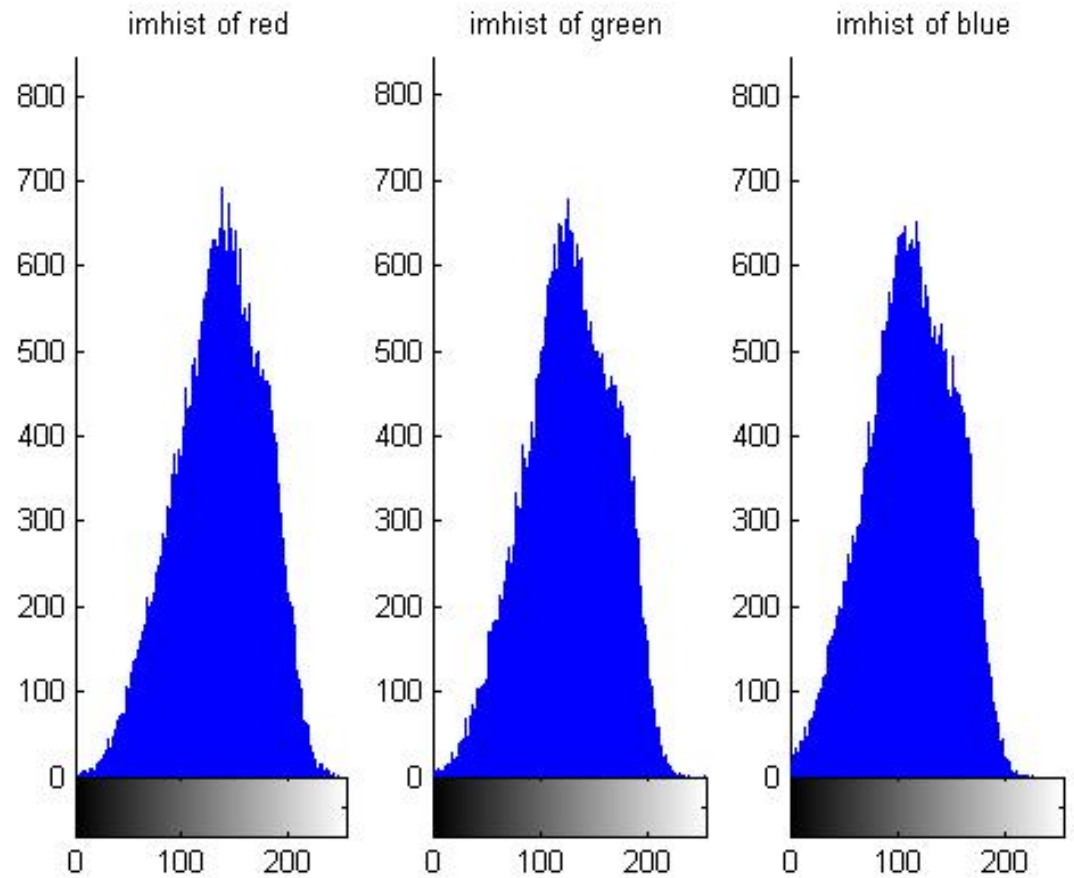

Figure 15. Histogram of encrypted baboon image.

From the histogram information, the gray value distribution of the three color channels has changed greatly. Three directions correlation before and after encryption are shown in Figure 16.
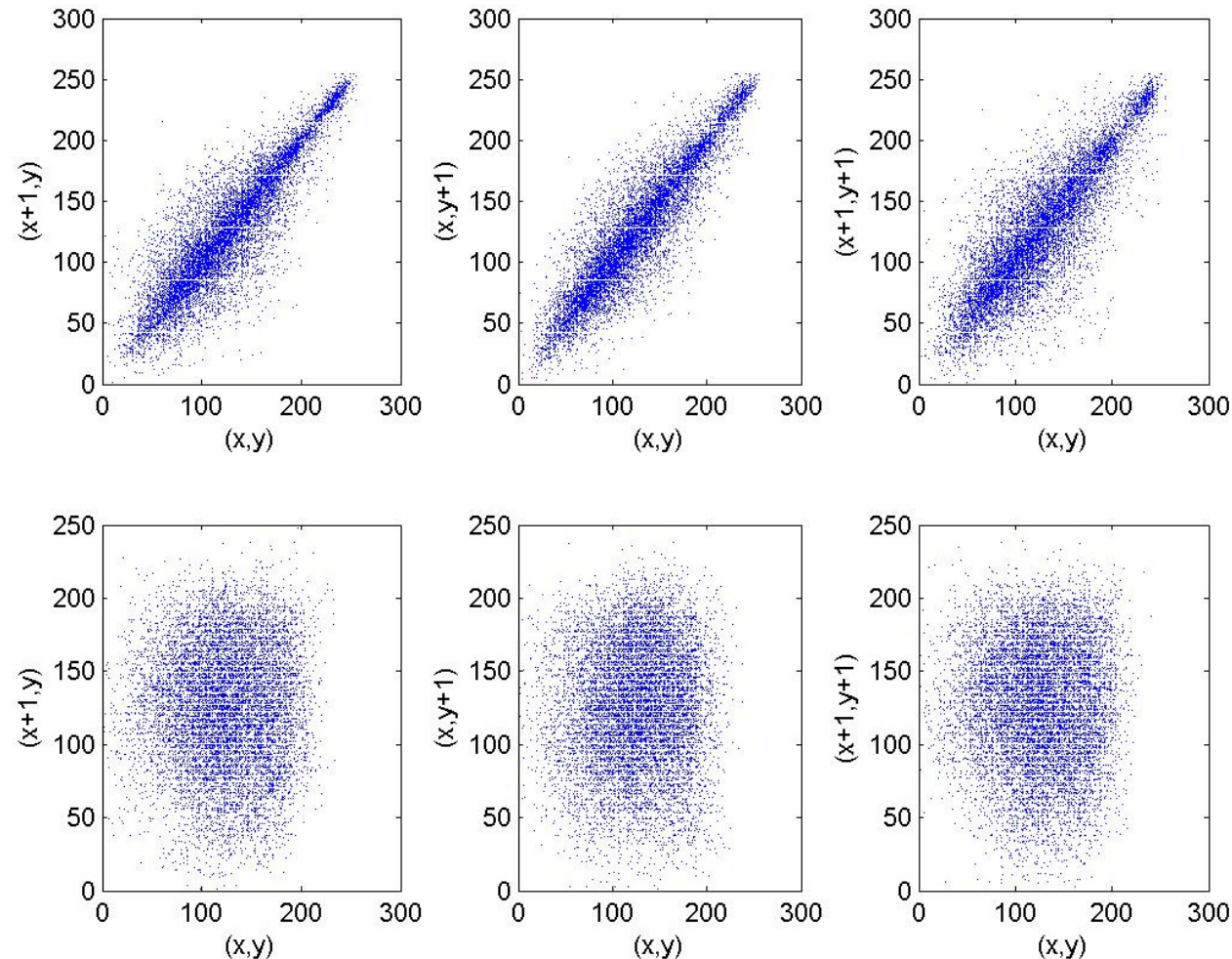

Figure 16. Correlation charts of three directions before and after encrypted baboon image.

The correlation between the three directions indicates the degree of correlation between them. As for the analysis of the ability to resist the difference attack, details are shown in Table 5 . 
Table 5. Baboon's information entropy and NPCR, UACI.

\begin{tabular}{ccccc}
\hline & Hm1 & Hm2 & NPCR & UACI \\
\hline baboon & 7.6701 & 7.39777 & 0.1602 & 0.0682 \\
\hline
\end{tabular}

For the encryption of animal images, the meaning is very important, which can well disrupt the information of a hunter's pursuit path and so on. Even if poachers obtain animal image information, it is difficult to obtain normal and real information. Information entropy can well express how much information the image contains. As long as there are some differences, it will increase many difficulties in cracking the content of image encryption.

\section{Conclusions and Discussion}

In view of the security in need of the network transmission image, the encrypted image is easily cracked by hackers or is difficult to completely restore the encrypted image. According to the features of each image, there are obvious changes in the main features before and after encryption. The decrypted image is inferior to the original image, and the value obtained is very small. After being converted into image format, the decryption effect is very good. The encryption and decryption algorithm proposed in this paper is simple and easy to implement, which not only reduces the computational complexity of the encryption algorithm, but also improves the security of the encryption algorithm. The information contained in the decrypted image and the original image had not changed much, which shows that the decryption effect is good. The validity of the proposed algorithm has yet to be verified in detail on large international datasets. The future research direction will introduce a modern intelligent algorithm, deep neural network and so on to encrypt or decrypt the image, or introduce a varied chaos system to protect the image. In the future, comprehensive modern intelligent algorithms may be introduced to encrypt and decrypt, or to introduce varied chaotic systems and convert them into the frequency domain to encrypt, so that the security of image transmission can be guaranteed.

Author Contributions: Conceptualization, B.W. and H.Z.; methodology, C.C.; software, C.C.; validation, C.C.; formal analysis, C.C.; investigation, C.C.; resources, C.C.; data curation, C.C.; writingoriginal draft preparation, C.C.; writing-review and editing, C.C.; visualization, C.C.; supervision, C.C.; project administration, C.C.; funding acquisition, C.C. All authors have read and agreed to the published version of the manuscript.

Funding: This research was funded by the Project of State Administration of Science, Technology and Industry for National Defence grant number: 20zg6108. And the APC was funded by the Project of State Administration of Science, Technology and Industry for National Defence.

Informed Consent Statement: Not applicable.

Conflicts of Interest: The authors declare no conflict of interest.

\section{References}

1. Li, X.; Zhou, C.; Xu, N. A secure and efficient image encryption algorithm based on DNA coding and spatiotemporal chaos. Int. J. Netw. Secur. 2018, 20, 110-120. [CrossRef]

2. Liu, H.; Jin, C. A color image encryption scheme based on arnold scrambling and quantum chaotic. Int. J. Netw. Secur. 2017, 19, 347-357.

3. Farwa, S.; Shah, T.; Muhammad, N.; Bibi, N.; Jahangir, A.; Arshad, S. An Image Encryption Technique based on Chaotic S-Box and Arnold Transform. Int. J. Adv. Comput. Sci. Appl. 2017, 8, 360-364. [CrossRef]

4. Jin, X.; Yin, S.; Li, X.; Zhao, G.; Tian, Z.; Sun, N.; Zhu, S. Color image encryption in YCbCr space. In Proceedings of the International Conference on Wireless Communications \& Signal, Yangzhou, China, 13-15 October 2016. [CrossRef]

5. Ni, Z.; Kang, X.; Wang, L. A novel image encryption algorithm based on bit-level improved Arnold transform and hyper chaotic map. In Proceedings of the IEEE International Conference on Signal and Image, Kuching, Malaysia, 17-20 September 2017; pp. 156-160. [CrossRef]

6. Hu, Y.; Xie, X.; Liu, X.; Zhou, N. Quantum Multi-Image Encryption Based on Iteration Arnold Transform with Parameters and Image Correlation Decomposition. Int. J. Theor. Phys. 2017, 56, 2192-2205. [CrossRef] 
7. Xu, L.; Gou, X.; Li, Z.; Li, J. A novel chaotic image encryption algorithm using block scrambling and dynamic index based diffusion. Opt. Lasers Eng. 2017, 91, 41-52. [CrossRef]

8. Vaish, A.; Kumar, M. Color image encryption using MSVD, DWT and Arnold transform in fractional Fourier domain. Opt. -Int. J. Light Electron Opt. 2017, 145. [CrossRef]

9. Chai, X.; Gan, Z.; Chen, Y.; Zhang, Y. A visually secure image encryption scheme based on compressive sensing. Signal Process. 2017, 134, 35-51. [CrossRef]

10. Song, L.; Yang, F.; Pan, G. Arnold transform and Gray code transformation of the dual scrambling algorithm to study. Comput. Appl. Softw. 2016, 33, 304-307.

11. Zhang, Z.; Li, Y. Tetrolet transform and SVD combination of blind detection robust digital watermarking embedding strategy. Comput. Eng. Sci. 2017, 39, 492-499.

12. Xie, G.; Su, B.H. Digital image encryption algorithm based on Arnold hierarchical cyclic transformation. Comput. Eng. Des. 2017, $38,1200-1204$

13. Zhang, Y.; Zhang, B. Image encryption algorithm based on Logistic chaotic system. Comput. Appl. Res. 2015, 32, 1770-1773.

14. Wang, Y.; Zhou, S. Image encryption algorithm based on fractional Chen's chaotic system. Comput. Appl. 2013, 33, 1043-1046. [CrossRef]

15. Gong, L.; Ceng, S.; Zhou, N. Color image encryption algorithm based on spectral cutting and two-dimensional Arnold transform Comput. Appl. 2012, 32, 2599-2602. [CrossRef]

16. Li, C.; Luo, G.; Li, C. Image encryption scheme based on skew tent chaos map and Arnold transform. Comput. Appl. Res. 2017, 87, 127-133. [CrossRef]

17. Li, S.; Fang, D.L. A color image watermarking algorithm based on Amold transform. Microelectron. Comput. 2017, 53-57.

18. Xiao, Z.; Zhang, H.; Chen, H. Zero watermarking for enhanced singular value decomposition and cellular neural network. Chin. J. Image Graph. 2017, 22, 288-296. [CrossRef]

19. Wu, G.C.; Baleanu, D.; Xie, H.P.; Chen, F.L. Chaos synchronization of fractional chaotic maps based on the stability condition. Phys. A Stat. Mech. Appl. 2016, 460, 374-383. [CrossRef]

20. Wu, G.C.; Baleanu, D. Discrete chaos in fractional delayed logistic maps. Nonlinear Dyn. 2015, 80, 1697-1703. [CrossRef]

21. Abdeljawad, T.; Banerjee, S.; Wu, G.C. Discrete tempered fractional calculus for new chaotic systems with short memory and image encryption. Optik 2020, 203, 163698. [CrossRef]

22. Wu, G.C.; Deng, Z.G.; Baleanu, D.; Zeng, D.Q. New variable-order fractional chaotic systems for fast image encryption. Chaos 2019, 29, 083103. [CrossRef]

23. Saeed, S.T.; Riaz, M.B.; Awrejcewicz, J.; Ahmad, H. Exact Symmetric Solutions of MHD Casson Fluid Using Chemically Reactive Flow with Generalized Boundary Conditions. Energies 2021, 14, 6243. [CrossRef]

24. Aneja, M.; Sharma, S. Numerical study of bioconvection flow of nanofluids using non-Fourier's heat flux and non-Fick's mass flux theory. Int. J. Mod. Phys. B 2019, 33, 1950376. [CrossRef]

25. Saeed, S.T.; Riaz, M.B. A fractional study of generalized Oldroyd-B fluid with ramped conditions via local \& non-local kernels. Nonlinear Eng. 2021, 10, 177-186. [CrossRef]

26. Ur Rehman, A.; Riaz, M.B.; Akgül, A.; Saeed, S.T. Dumitru Baleanu Heat and Mass Transport Impact on MHD Second-Grade Fluid: A Comparative Analysis of Fractional Operators; Wiley: Hoboken, NJ, USA, 2021; Volume 50, p. 6. [CrossRef]

27. Abdeljawad, T.; Riaz, M.B.; Saeed, S.T.; Iftikhar, N.I. MHD Maxwell Fluid with Heat Transfer Analysis under Ramp Velocity and Ramp Temperature Subject to Non-Integer Differentiable Operators. Comput. Model. Eng. Sci. 2021, 126, 821-841. [CrossRef] 